\title{
Short-Range C-Band Noise Radar for Meteorological Application
}

\author{
Lukasz Maslikowski and Krzysztof Kulpa \\ Warsaw University of Technology \\ Institute of Electronic Systems \\ Nowowiejska, 15/19, 00-665 Warsaw, POLAND \\ Email: 1.maslikowski@stud.elka.pw.edu.pl
}

\author{
Dmitriy Glushko and Felix J. Yanovsky \\ National Aviation University \\ Electronics Department \\ Prospekt Komarova 1, 03-680 Kiev, UKRAINE \\ Email: yanovsky@nau.edu.ua
}

\begin{abstract}
This paper concerns a ground-based continuouswave noise radar system that was built from Commercial-OffThe-Shelf measurement equipment (Vector Signal Analyser, Arbitrary Waveform Generator, amplifiers, antennas) and operated at $5.6 \mathrm{GHz}$ in the $\mathrm{C}$-band. This electronic system was tested as a meteorological radar in the experiment devoted to atmospheric precipitation sensing. The result of the processing is a set of Range-Doppler plots containing visible echo of rain, based on which its intensity can be calculated. There are the system setup, signal processing and results described in the paper.
\end{abstract}

Keywords-noise radar, software radar, solid state radar, signal processing, meteorological radar.

\section{INTRODUCTION}

During many years meteorological objects and phenomena were studied using conventional pulse radars and Doppler radars (pulse [1] or CW FM [2]). Recently polarimetric Doppler radar [3] and Doppler-polarimetric radar is considered as a powerful instrument for remote sensing of the atmosphere [4]. The simplest but perhaps one of the most important characteristics of precipitation is the intensity of rain or snow. It is well-known that rain intensity can be estimated from measured Radar Reflectivity Factor (RRF) $Z$ (measured at single polarization, for example at horizontal polarization $Z_{h}$ ) which is directly proportional to the average power of receiving signal. One of disadvantages related with this way of rain rate estimation is the necessity to perform absolute measurements of the reflected power that requires complicated calibration and radar parameters stabilization. Otherwise the results become inaccurate.

Dual-polarization radar, which is naturally a multiparameter system, offers some new parameters that are related with rain rate. First of all such parameters are differential reflectivity $Z_{D R}$ and specific differential phase $K_{D P}$. Differential reflectivity can be defined as $Z_{D R}=10 \log \left(Z_{h} / Z_{v}\right)$, where $Z_{h}$ and $Z_{v}$ are RRF of the same resolution volume measured at two orthogonal polarizations, say, horizontal (h) and vertical (v) correspondingly. Specific differential phase is defined as $K_{D P}=\left[\phi_{D P}\left(r_{2}\right)-\phi_{D P}\left(r_{1}\right)\right] / 2\left(r_{2}-r_{1}\right)$. Here $\phi_{D P}=\phi_{h}-\phi_{v}$ is a differential phase, and $\phi_{h}$ is the phase of the horizontally-polarized pulse at a given point in the propagation path, $\phi_{v}$ is the phase of the verticallypolarized pulse at the same point in the propagation path, $r_{1}$ and $r_{2}$ refer to measurements at range 1 and range 2 from the radar, $r_{2}>r_{1}, \phi_{h} \geq 0, \phi_{v} \geq 0$. This definition shows that differential phase $\phi_{D P}$ is simply the difference in phase between the horizontally- and vertically-polarized pulses at a given range along the propagation path. Naturally, $\phi_{D P}$ will increase with range from the radar, so we can take the range derivative over $\Delta r=r_{2}-r_{1}$ to determine where along the propagation path phase changes are occurring. This derivative is called the specific differential phase, or $K_{D P}$. In the denominator a multiplier 2 appears at $\Delta r=r_{2}-r_{1}$ because there is a phase shift on both, the outbound trip and the return trip.

Detailed analysis and physical explanation of the relationships between above-mentioned measurable variables and rain rate $R$ can be found, for example, in [3]. Physically based radar rain rate estimation algorithms can be broadly classified into four categories, namely, $R(Z), R\left(Z, Z_{D R}\right), R\left(K_{D P}\right)$ and $R\left(K_{D P}, Z_{D R}\right)$. Each of these algorithms has advantages and disadvantages. Important to mention that polarimetric parameters $Z_{D R}$ and $K_{D P}$ are relative parameters, so they do not suffer from problems with calibration that are characteristic for absolute value measurements. Of course, suitable engineering implementation of rain rate algorithm can be done using statistical approach or on the basis of fuzzy logics or/and neural network. However this is beyond the scope of this paper.

Thus, narrowband Doppler radars both pulse and CW FM, including radars with polarization diversity, became operational tools for remote sensing of meteorological objects. Nevertheless, they have some disadvantages. The range cell size depends on the length of the pulse or bandwidth, so it is difficult to achieve very good resolution in range with adequate power and velocity resolution. Another problem is related to ambiguous returns that cause a severe limitation when studying widely distributed targets as weather objects. One of the first mentions of noise radar for meteorological application was made in [5]. Meteorological application of UWB including noise waveform was discussed in [6]. In [5] it was shown that the frequency band of transmitting signal and the filter band in meteorological noise radar can be set and controlled independently; thus, there is possible to control simultaneously both resolution on distance and velocity. Moreover, the transmitting signal is not a periodical one, so there are no ambiguities in distance measuring, that is, it is possible to set parameters to provide unambiguous velocity range which is interesting for given application of the noise radar. That means that noise radar can measure very wide Doppler spectra. The last advantage is especially important for 
the case of Doppler-polarimentric measurements because they need measurable information on the spectrum itself but not only the spectrum width [7].

Nowadays, a noise radar can be implemented as a softwaredefined system. Due to growing computational powers, even radar with large bandwith and resulting abundant stream of digital data can be implemented to work in real-time. The restrictions on the frequency band or the central frequency of the transmitter depend mainly on the analogue front-end and the ADC capabilities. Experimental implementation of noise sounding waveform for sounding the atmosphere was checked using acoustic radar (sodar) as was described in [8]. The noise radar seems to be an interesting option to implement a weather radar. Taking into account the experience on noise radar development accumulated in institutions like Warsaw University of Technology [9] or Institute for Radiophysics and Electronics, NAS of Ukraine [10] we can say that the technology is mature enough to be used in practice. In addition, an important advantage of the noise radar is that it is highly immune to noise and unintentional jamming which can be a significant problem as the occupation of radio frequencies increasingly grows. The purpose of this paper is to describe an experiment on atmospheric precipitation sensing with a $\mathrm{C}$ band ground-based CW noise radar.

\section{SySTEM SETUP}

The system constructed in order to carry out an experiment was a software defined noise radar. It was built from COTS (Commercial-Off-The-Shelf) elements. It used an Arbitrary Waveform Generator (AWG - NI-PXIe 5673) as the source of the sounding waveform and a an Vector Signal Analyser (2 channel VSA - NI-PXIe 5663) as the receiver. The transmitted waveform was a realization of pseudo-noise white, complex Gaussian noise generated in Matlab environment and uploaded to the memory of the AWG. It was synthesized in the baseband at a sampling clock frequency of $10 \mathrm{MHz}$ and modulated to the desired carrier of $5.6 \mathrm{GHz}$. The waveform contained $10 \mathrm{MS}$ repeated in a continuous loop. Due to the fact that the time of a single integration was set to 1 second and covered $10 \mathrm{MS}$, there were no ambiguities in the resultant crossambiguity functions. In a ready-made noise radar the waveform should not rather be periodic, however for this kind of experiment it did not bring any inconveniences but simplified the measurement itself. The generator offered maximum output power of $5 \mathrm{dBm}$ so an additional power amplifier on the transmitting site was needed. As a result the power supplied to a commercially available 5.6 $\mathrm{GHz}$ grid antenna was about $20 \mathrm{dBm}$. A noise radar needs a reference signal for correlational range compression to be performed. In the given case such signal was captured by the second, time-synchronized and phase-coherent channel of the VSA. This provided also the synchronization between transmission and reception that needs particular hardware support in conventional pulse radars. To improve the SNR, a bandpass filter and low-noise amplifiers were put after the receiving antenna. In order to evaluate the power balance in the system, a measurement in calibration connection with attenuator instead of antennas was also carried out. The diagram of the system setup is presented in Fig. 1.

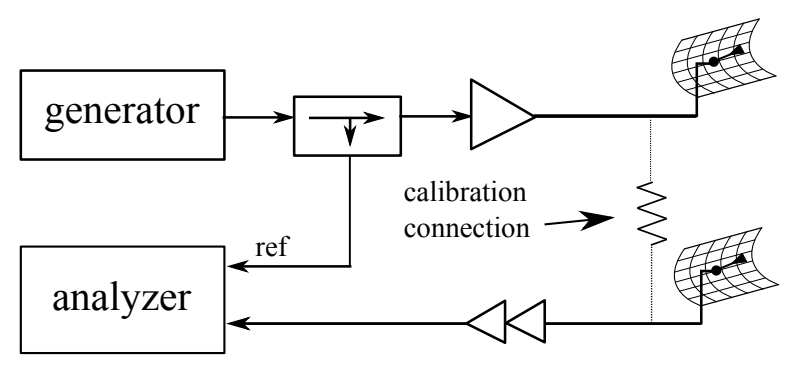

Fig. 1. A diagram of the system setup.

\section{SIGNAL PROCESSING}

All the signal processing of the received signals was done offline in the MATLAB environment. It could be divided in two main steps. The first was cancellation of the direct-path interference resulting from $\mathrm{CW}$ operation mode. In case of the described experiment, the echo signal was at least $70 \mathrm{~dB}$ weaker than the direct one. For the bandwidth-time product of $10^{7}$ samples, the echoes of such power are covered under residual fluctuations resulting from the strongest signal. In order to suppress the direct signal together with the disturbances it introduces to the crossambiguty function, a block lattice filter was used [11]. It reduced the residual fluctuations level by approximately $30 \mathrm{~dB}$, which revealed the useful echoes. Then range-Doppler correlation between the reference and surveillance signal was done to obtain the crossambiguity function. The surveillance signal was correlated with Doppler-shifted copies of the reference signal with removed interferences. The results were then normalized to the system reference power level. It was obtained from range-Doppler plot (with no lattice filtering) calculated for data collected in calibration setup (with a known attenuator connecting $\mathrm{Rx}$ and $\mathrm{Tx}$ antenna cables).

With the knowledge of antenna gain and other radar specifications the calculation of precipitation intensity can be done. Different algorithms can be used for this purpose. As an example, in this paper we use the simplest algorithm which was mentioned in the introductory part of the paper. Radar equation in case of meteorological target observation can be written as [12]:

$$
P_{r}=Z \frac{\pi^{4} P_{t} G_{t} G_{r} c|K|^{2} k_{f i l l}}{128 \lambda^{2} B L_{0} r^{2}}
$$

where $P_{r}$ and $P_{t}$ are received and transmitted powers, $G_{r}$ and $G_{t}$ are gains of receiving and transmitting antennas, $\lambda$ is the wavelenght of the carrier, $B$ is the spectrum width of the sounding noise waveform, $c$ is the lightspped, $L_{0}$ is a coefficient of losses, $|K|^{2}$ is a coefficient depending on the material of scatterers (e.g. for water $|K|^{2}=0.93$, for ice $\left.|K|^{2}=0.19\right), k_{f i l l}$ is a fill factor that shows which part of the resolution volume is filled with scatterers $\left(0 \leq k_{\text {fill }} \leq 1\right)$, $r$ is a distance between the radar and the resolution volume under observation (radar range), $Z$ is radar reflectivity factor which shows the reflection power of the object and depends on the microstructure of the resolution volume under observation.

Let us assume $k_{f i l l} \approx 1$ (this is practically always true in case of high radar resolution, that is small resolution volume), combine all radar specifications, except the transmitting power, 


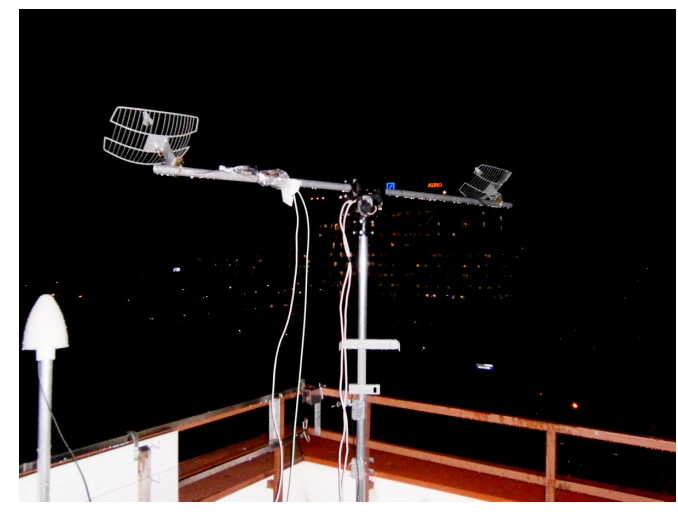

Fig. 2. Antennas during the measurement.

as a dimensional factor $\Xi$, and solve equation (1) regarding $Z$. Then we receive:

$$
Z=\frac{\Xi r^{2}}{|K|^{2}} \cdot \frac{P_{r}}{P_{t}},
$$

where $\Xi=\left(128 \lambda^{2} B L_{0}\right) /\left(\pi^{4} G_{t} G_{r} c\right)$.

Thus, radar reflectivity factor $Z$ can be estimated from measured $P_{r} / P_{t}$ ratio with assumption of $|K|^{2}$ value (say water, ice, snow) and known parameters of the radar $(\Xi)$.

In turn, radar reflectivity factor is related with different important meteorological parameters (rain rate, water content, etc). In case of rain, the intensity of rainfall (or rain rate) $R$ can be estimated from:

$$
R(Z) \approx \alpha Z^{\beta}\left[\mathrm{mm} \cdot \mathrm{h}^{-1}\right]
$$

where $Z$ is in units of $\left[\mathrm{mm}^{6} \cdot \mathrm{m}^{-3}\right]$.

Parameters $\alpha$ and $\beta$ in the empirical expression (3) depend on different factors. The range of these parameters reported in the literature is very large. In moderate latitudes one can use $\alpha=0.041$ and $\beta=0.625$ (taken from $Z \approx 200 R^{1.6}[13]$ ). For US weather radar WSR-88D according to [3] $R_{\mathrm{WSR}}(Z) \approx$ $0.017 Z^{0.714}$.

Of course, application of polarimetric measurements of $Z_{D R}$ and $K_{D P}$ can make results more accurate, however the purpose of this work is just to show possibility of meteorological measurements with a $\mathrm{CW}$ noise radar.

\section{MEASUREMENT CAMPAIGN AND ITS RESULTS}

The measurement campaign was conducted in October 2012 at the balcony of the DSP Laboratory of Warsaw University of Technology at the $5^{\text {th }}$ floor over the ground. During rain observation two grid antennas with directivity of $25 \mathrm{dBi}$ were mounted on a rotator. The rain intensity was maximum $25 \mathrm{dBz}$ (according to weather radar data available online). Several measurements including azimuth and elevation scans were done. Fig. 2 shows a photo of the measurement setup while in the Fig. 3 an exemplary result for rain observation is presented. During this measurement antennas were directed approximately at $45^{\circ}$ in elevation. It can be seen that at further distances and thereby bigger heights, the raidrops moved faster in the radial direction.

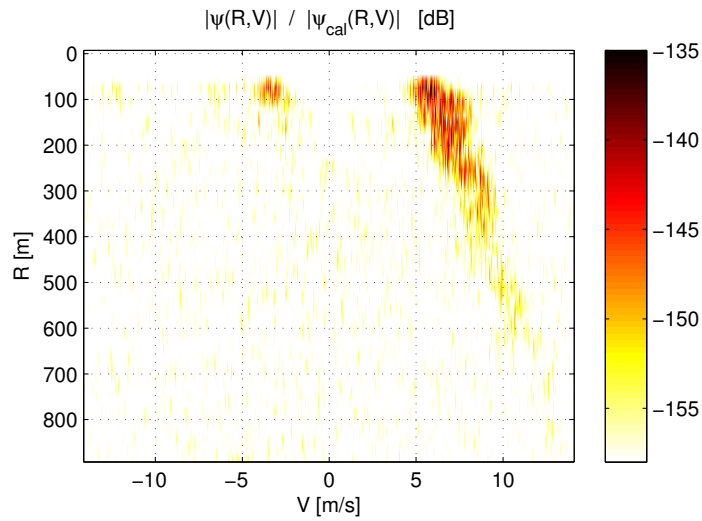

Fig. 3. Echo of the rain visible at positive Doppler velocities.

\section{CONCLUSIONS}

The paper has shown the potential of use of a low power, COTS-based noise radar demonstrator as a source of weather data. In the future the authors plan to perform multichannel, polarimetric trials with a noise radar to obtain data that can make full use form accurate methods of recalculation of collected results into meaningful meteorological quantities.

\section{REFERENCES}

[1] R. Doviak and D. Zrnic, Doppler Radar and Weather Observations. San Diego: Academic Press, 1993.

[2] L. Ligthart and L. Nieuwkerk, "An X-band solid-state FM-CW weather radar," Radar and Signal Processing, IEE Proceedings F, vol. 137, no. 6, p. 41, dec 1990 .

[3] V. N. Bringi and V. Chandrasekar, Polarimetric Doppler Weather Radar: Principles and Applications. Cambridge, UK: Cambridge University Press, 2001.

[4] F. Yanovsky, H. Russchenberg, and C. Unal, "Retrieval of information about turbulence in rain by using doppler-polarimetric radar," Microwave Theory and Techniques, IEEE Transactions on, vol. 53, no. 2, pp. 444-450, Feb. 2005.

[5] F. J. Yanovsky, "Potential of a noise radar for meteorological applications," in First International Workshop on the Noise Radar Technology (NRTW 2002), Sep. 2002, pp. 195-199.

[6] F. Yanovsky, V. Kharchenko, and L. Ligthart, "Wideband radar remote sensing of the atmosphere: a data interpretation problem," in Ultrawideband and Ultrashort Impulse Signals, 2004 Second International Workshop, Sept. 2004, pp. $42-47$

[7] F. Yanovsky, "Phenomenological models of doppler-polarimetric microwave remote sensing of clouds and precipitation," in Geoscience and Remote Sensing Symposium, 2002. IGARSS '02. 2002 IEEE International, vol. 3, june 2002.

[8] R. Sinitsyn and F. Yanovsky, "Signal processing algorithms for ultrawideband noise radar," in Proc. International Radar Symposium IRS 2007, Sep. 2007, pp. 83-88.

[9] L. Maslikowski, M. Malanowski, K. Kulpa, and C. Contartese, "Preliminary results of ground-based noise SAR experiments," in Proc. EUSAR 2010, June 2010, pp. 596-599.

[10] K. Lukin, P. Vyplavin, V. Palamarchuk, O. Zemlyaniy, V. Kudriashov, and S. Lukin, "Capabilities of noise radar in remote sensing applications," in Advances in Radar and Remote Sensing (TyWRRS), 2012 Tyrrhenian Workshop on, Sept. 2012, pp. 10-17.

[11] K. Kulpa and Z. Czekala, "Ground clutter suppression in noise radar," in Int. Conference RADAR 2004, Oct. 2004, pp. 236-240.

[12] F. Yanovsky, Meteorological and Navigation Radar Systems of Aircraft (in Ukrainian). Kiev, Ukraine: NAU Press, 2003.

[13] V. Stepanenko, Radar in Meteorology (Radiometeorology) (in Russian). Leningrad, form. USSR: Gidrometeoizda, 1966. 